\title{
Multi-material Anisotropic Friction Wheels for Omnidirectional Ground Vehicles
}

\author{
Genya Ishigami $^{* 1}$, Jim Overholt ${ }^{* 2}$, Karl Iagnemma ${ }^{3}$ \\ *1 Institute of Space and Astronautical Science, Japan Aerospace Exploration Agency \\ 3-1-1 Yoshinodai, Sagamihara, Kanagawa 252-5210 JAPAN \\ *2 United States Army TARDEC \\ 6501 E. 11 Mile Road, Warren, MI 48397, USA \\ *3 Laboratory for Manufacturing and Productivity, Massachusetts Institute of Technology \\ 77 Massachusetts Ave., Cambridge, MA 02139 USA
}

\begin{abstract}
:
In this paper, a novel omnidirectional vehicle with anisotropic friction wheels is presented. The proposed wheel has a series of bendable "nodes" on its circumference, each of which is made of two materials with differing friction properties: one material exhibits high friction, and the other exhibits low friction. The high friction section of the node generates a high traction force, while the low friction section enables the wheel to passively slide. The wheels are arranged such that the robot wheel exhibits high traction in its driving direction (much like a conventional tire), but low traction when sliding laterally. Due to this "anisotropic friction" property, the proposed wheel enables a vehicle to realize omnidirectional motion (i.e. the vehicle can move any direction within the plane-forward, back, or laterally). While many other omnidirectional wheel drives exist, the proposed wheel is simpler than any other existing design because the wheel is composed of a single, moldable element. This paper summarizes the design of the proposed wheel and presents a comparison between a small omnidirectional vehicle that uses the proposed wheel and an omnidirectional vehicle that uses conventional wheels.
\end{abstract}

\section{INTRODUCTION}

An omnidirectional vehicle is able to kinematically move in any planar direction regardless of its current pose. This is in contrast to typical steered wheeled vehicles, which must travel along arcs, and thus have difficulty performing complex maneuvers. Omnidirectional vehicles are commonly applied in many practical areas: mobile robotic bases for research, materials handling vehicles (i.e. fork trucks) for logistics, and wheelchairs.

To date, a wide variety of wheels have been developed that allows a vehicle to realize omnidirectional motion. Roller wheel designs [1]-[3], as shown in Fig. 1, employ a series of slender rollers along the circumference of a wheel that are mounted perpendicular to the wheel rotational direction. The wheel generates thrust in the wheel's rotational (longitudinal) direction and passively slides in the lateral direction using the slender rollers.
Omnidirectional motion of vehicle is obtained by orienting several of these wheels (usually, three or four) in different directions (Fig.2). For additional information, see [4][5]. The common design element of these wheels is that they possess a single wheel with a large ("major") diameter, which contains many smaller diameter wheels with axes of rotation that are skew to the major axis.
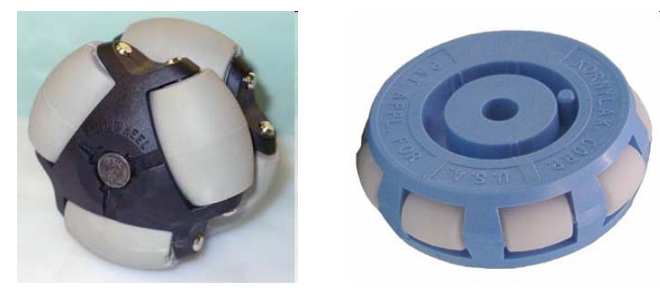

Fig.1 Examples of roller wheels (Left: Omniwheel, Right: Transwheel ${ }^{\circledR}$, from [3])
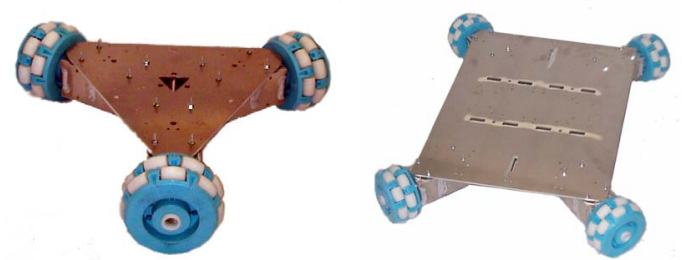

Fig.2 Examples of omnidirectional vehicle with roller wheels (from [6])
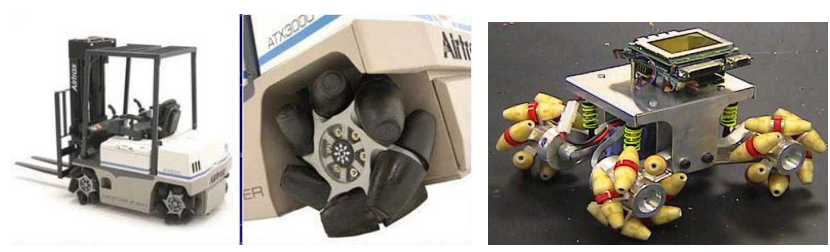

Fig. 3 Examples of vehicles using Mecanum wheels (from [7] and [8])

Mecanum wheels are similar to roller wheels in that they employ rollers along the outer edge of a wheel; however, the rollers are aligned at 45 degrees to the plane of the wheel to produce angular contact forces with the ground. Vehicles equipped with four mecanum wheels, as shown in Fig. 3, can produce omnidirectional motion. 
While the vehicles can move forward and backward as conventional wheels do, they can also move laterally by rotating the front and rear wheels in opposite directions.

A drawback of these wheel designs is that they are complex, with numerous roller, axle, and bearing elements. The novel wheel described in this paper is able to realize omnidirectional motion by exploiting the same “driving and sliding” property utilized by conventional omnidirectional drive wheels. The novelty and utility of the invention derives from the fact that the wheel is a single component, with two distinct materials. Thus the proposed wheel is less complex, more robust (due to fewer moving components), and potentially less expensive to produce than conventional omnidirectional wheels.

This paper is organized as follows: Section 2 describes the design of the proposed wheel and mechanism of the anisotropic friction realized by the wheel. Section 3 presents a prototype of an omnidirectional vehicle using the proposed wheels. A comparison of the proposed wheel and conventional wheels are also described.

\section{OMNIDIRECTIONAL WHEEL WITH ANISOTROPIC FRICTION}

\subsection{Design Concept}

The proposed wheel has a large ("major") diameter with a series of bendable "nodes" on its circumference (see Fig. 4). Each node is composed of two materials with differing friction property. A high friction material is present in a narrow strip on the surface of the node, with the strip oriented parallel to the wheel face (i.e. perpendicular to the axis of rotation of the wheel). The high friction material can be imparted to the node through various manufacturing processes, including molding and deposition, or by adhesion or fastening. In the prototype shown in Fig. 4, the high friction material was imparted in a rapid prototyping process - thus the wheel was "printed" from multiple materials in a single process. During wheel rotation, the high friction strip contacts the ground and generates a traction force in the vehicle's longitudinal direction.

A node's side faces (i.e. the regions outside the strip) are composed of a material with low friction properties. Also, the node itself is designed in such a way that the connection to the major diameter (the "root") is somewhat flexible. Therefore, when the wheel is pushed laterally while contacting the ground, the node deforms slightly, which causes the low friction material to contact the ground (see Fig. 5). This causes the vehicle to slide. This phenomenon is described further in Section 2.3.

When multiple wheels (ideally four) are arranged as shown in Fig. 6, wheels in orthogonal directions can be driven to generate traction forward, backward, or laterally. The driven wheels experience only modest resistance from sliding wheels. Thus, by exploiting the anisotropic friction property described here, a vehicle with the proposed wheels can realize omnidirectional motion.

\subsection{Omnidirectional Wheel}

A CAD design and prototype of the proposed wheel for omnidirectional vehicle is shown in Fig.4. The prototype has a dimension of $49.0 \mathrm{~mm}$ (diameter) and $18.5 \mathrm{~mm}$ (width), and weighs $15.0 \mathrm{~g}$. The wheel consists of wheel rim made of low friction material (83 Shore Scale D, gray-colored part in Fig.4), a thin strip of high friction material (27 Shore Scale A, orange-colored part), and a series of bendable nodes. The wheel is fabricated as a single-piece component using 3D printing technology [9], and it is possible to directly mount the wheel to a drive actuator.

The nodes should generally be closely and evenly spaced so that the motion resulting from the wheel is smooth and not bumpy in the vertical direction. In the prototype, two parallel rows of nodes were employed, with the rows slightly offset from one another, to increase smoothness of travel. In principle, three or more rows of nodes could be employed. In the current prototype, the total number of spikes is 32 aligned in dual lines, each half of which has 11.25 degrees of phase difference.
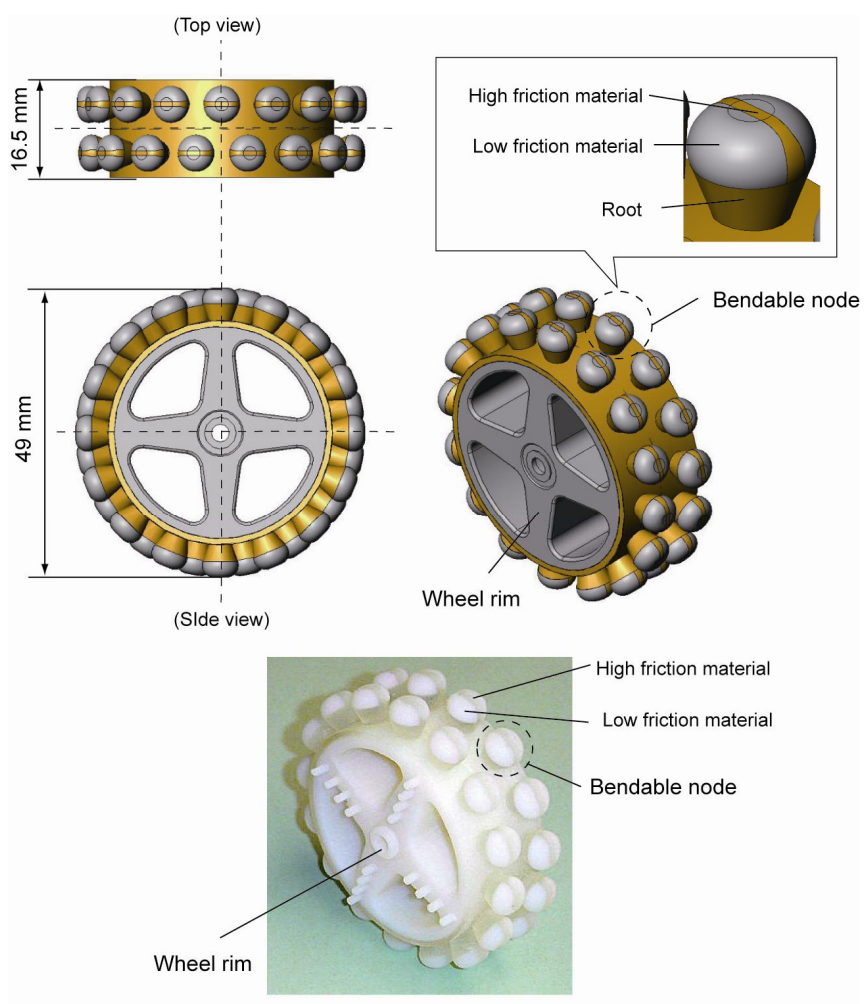

Fig.4. CAD model and prototype of the multi-material anisotropic friction wheel for omnidirectional vehicle 


\subsection{Bendable Node}

Omnidirectional motion of a vehicle with the proposed wheel is achieved via the anisotropic friction properties of the nodes. The nodes should have a high friction strip to grip the ground and generate traction forces, just as conventional pneumatic tires do. The nodes should also have low friction side faces that will contact the ground when the node bends at the root, so that the wheel can slide, resulting in lateral motion of the vehicle. The root is designed such that a nominal load on the wheel will cause the root to deflect enough that the face of the node (i.e. the point of contact with the ground) shifts from the high friction strip to the low friction area.

The initial concept of using an anisotropic material for anisotropic friction was first presented in [10], in the context of an inchworm-like mobile robot. There, a planar "foot" with anisotropic was used in a sliding manner. The main difference between the proposed wheel and the foot reported in [10] is that 1 ) the device reported in [10] is a planar mechanism and not a wheel, and 2) the device of [10] cannot be used in any obvious manner to achieve omnidirectional motion.

It should be noted that the particular design of the node in Fig. 4 is only one example of a set of possible designs that could exploit the principle of anisotropic friction for omnidirectional wheeled vehicles. The node could be designed in slightly different geometries to realize different load/deflection properties, which would affect vehicle motion. Also, the geometry of the high friction strip could potentially be modified somewhat while still maintaining omnidirectional motion properties.

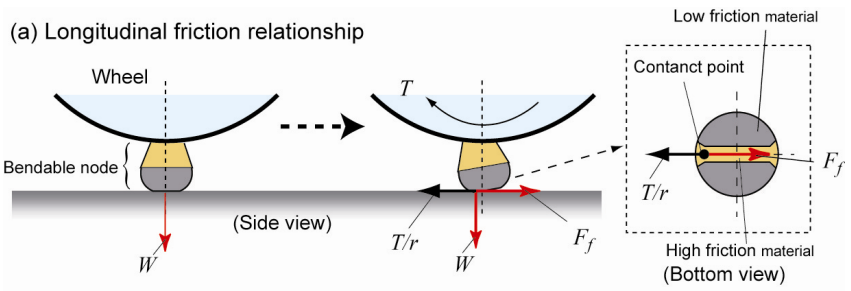

(b) Lateral friction relationship

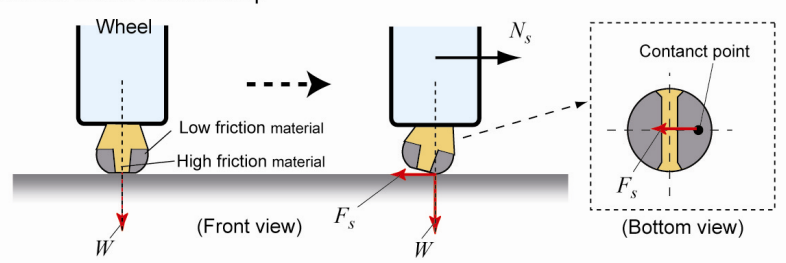

Fig.5. Anisotropic friction mechanics of the bendable node

\subsection{Anisotropic Friction Mechanics}

Fig.5 illustrates the mechanics of the anisotropic friction properties realized by the proposed wheel.

When the wheel is at rest, under load from the wheel and robot (and possible a payload), the high friction strip contacts the ground, since the high friction strip lies directly beneath the wheel (i.e. at "bottom dead center").

When a driving torque $T$ is applied to the wheel (Fig. 5(a)), the node bends due to low flexural stiffness at the root. Then, the high friction strip (the orange colored region in the bottom view) contacts the ground, generating a friction force $F_{f}=\mu_{h} W$. The material for high friction strip is chosen such that the friction coefficient $\mu_{h}$ is high, and therefore the driving force $F_{f}$ is large.

When a force $N_{s}$ is applied to the wheel in lateral direction (Fig. 5(b)), for example by forces caused by orthogonally-oriented driven wheels on the vehicle, the node bends laterally due to low flexural stiffness at the root. Then, the low friction region of the node contacts the ground and the high friction strip does not. In this scenario, the wheel generates a friction force $F_{s}=\mu_{l} W$. The material for low friction region is chosen such that the friction coefficient $\mu_{l}$ is low, and therefore the sliding force $F_{s}$ is small.

Assuming a vehicle employing four wheels in an orthogonally-oriented configuration (see Fig. 6), the net traction force $N_{s}$ generated by two wheels (i.e. right and left) is:

$$
N_{s}=2 F_{f}=2 \mu_{h} W
$$

Then, comparing $N_{s}$ to the sliding forces $F_{s}\left(=\mu_{l} W\right)$ due to the low friction material of the other two wheels (i.e. front and rear):

$$
N_{s}-2 F_{s}=2 \mu_{h} W-2 \mu_{l} W=2 W\left(\mu_{h}-\mu_{l}\right)
$$

The properties for high/low material are chosen such that they have a relationship as $\mu_{h}>\mu_{l}$. Therefore, from Eq. (2), $N_{s}>F_{S}$ is realized, and the vehicle can achieve omnidirectional mobility.

Comparing the mechanics of omnidirectional motion for a vehicle with conventional wheels to a vehicle with the proposed wheels, a vehicle with conventional wheels realizes an anisotropic friction effect through the use of slender rollers, as noted in Section 1. On the other hand, the proposed wheel exploits distinct friction properties of two distinct materials, and thus "tribologically" achieves an anisotropic friction effect.

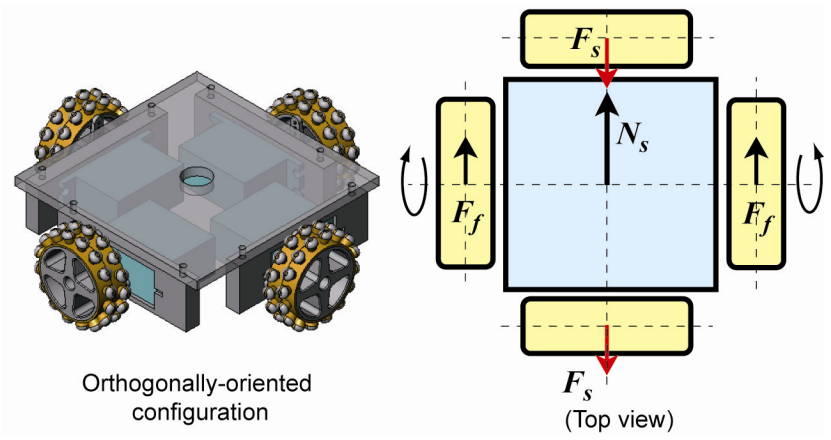

Fig.6. Vehicle in orthogonally-oriented configuration 
3. PRototype of OMNIDIREctional Robot AND EXPERIMENTAL TEST

\subsection{Omnidirectional Robot Prototype with Proposed Wheel}

Fig. 7 shows a physical prototype of an omnidirectional mobile robot that employs four of the proposed wheels in an orthogonally-oriented configuration. The dimensions of the robot are $159 \mathrm{~mm} \times 159 \mathrm{~mm}$, and weighs $690.0 \mathrm{~g}$. Each proposed wheel is actuated by servomotors. Power for the motors is supplied by four AA batteries. The robot is operated by an on-board micro controller, which sends drive signals to each servo to execute predefined omnidirectional motions (i.e. forward, back, or laterally, resulting in square motion, diagonal motion and others).

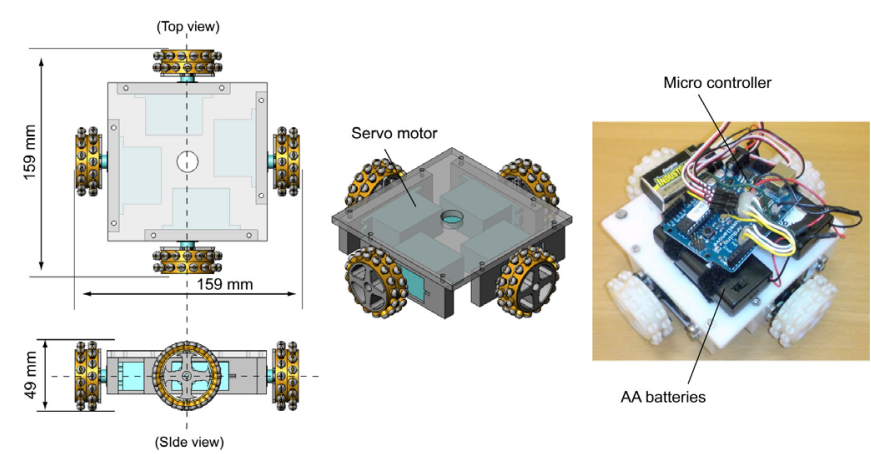

Fig.7. Prototype of the omnidirectional mobile with proposed wheel

\subsection{Performance Test}

Two experimental tests, omnidirectional mobility test and traction performance tests were conducted to study the motion performance of the proposed wheel. In the tests, two conventional omnidirectional wheels are used for performance comparison: Omniwheel and Transwheel ${ }^{\circledR}$, as shown in Fig.1. The outer diameter of these wheels is the same as the proposed wheel.

\subsubsection{Omnidirectional Mobility}

In these tests, the vehicle is controlled to repeatedly move along a square (i.e. right, forward, left, and back) at a fixed wheel velocity for a fixed amount of time, and the distance traveled is measured using camera images captured from above. Then, the omnidirectional mobility of the wheels (the proposed wheel, Omniwheel, and Transwheel) is compared based on the distance traveled.

The snapshots of the test are shown in Fig.8. The vehicle with the proposed wheels can achieve omnidirectional motion, and the distance traveled is $92.8 \%$ of that with Transwheel, and $99.0 \%$ of that with Omniwheel. This performance is likely to vary depending on the material that the vehicle is traveling on, since the friction coefficient is material dependent. However this test serves as a proof of concept of the utility of the proposed wheels.

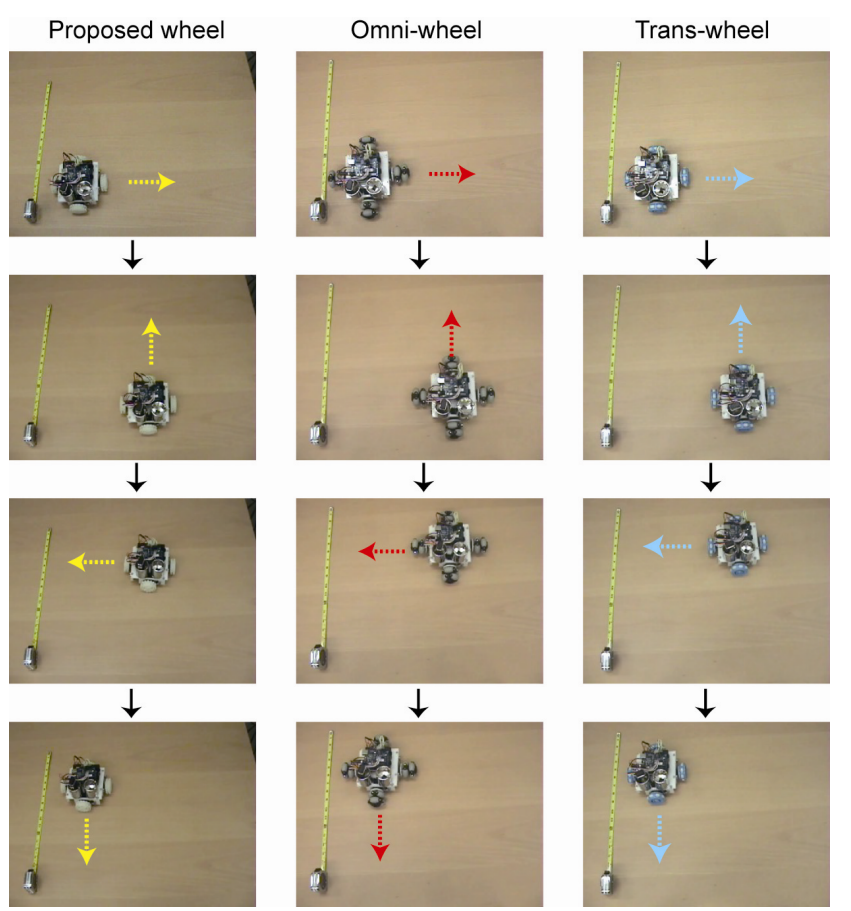

Fig.8. Omnidirectional mobility test

\subsubsection{Traction Performance}

The slope climbing test was conducted to evaluate the traction performance of the proposed wheel. In this test, the test bed is controlled to maintain its wheel driving velocity, and measure the slope angle $\theta_{\mathrm{s}}$ at which the test bed stalls. In this case, the maximum traction force generated by the wheels is equal to the tangential component of the vertical load of the test bed, namely $W \sin \theta_{\mathrm{s}}$.

Fig.9 shows the snapshots of the traction performance test. The slope surface is plywood. From the results, the proposed wheel generates traction force $\left(=W \sin \theta_{\mathrm{s}}\right)$ approximately 3.2 times larger than that of Transwheel, and 2.1 times larger than Omniwheel. The conventional ones are made of only low friction material, and because of this, the friction coefficient in longitudinal direction of wheel becomes relatively low, resulting in the low traction force. However, the proposed wheel has bendable node with high friction materials, which can stick to the ground and generate large traction force. This high traction performance of the proposed wheel is significant improvement over the conventional wheels.

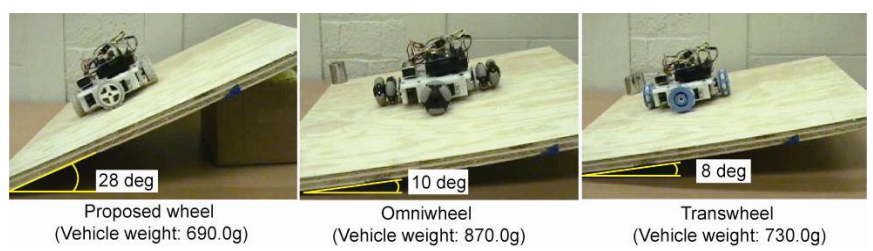

Fig. 9: Traction performance test 


\section{CONCLUSION}

In this paper, a novel design of wheel for omnidirectional vehicle utilizing anisotropic friction properties has been described. The proposed wheel has a series of bendable nodes on its circumference, the surface of which is covered with materials having differing friction property.

From the experimental results presented in Section 3.2, it has been confirmed that the vehicle with the proposed wheels has similar omnidirectional motion characteristics to a vehicle with conventional omnidirectional wheels. The proposed wheel can also grip to the ground with its high friction property, enabling the vehicle to generate much larger traction force than conventional wheels do. Thus, a significant improvement by the proposed wheel over the conventional omnidirectional wheels is the high traction performance while having equivalent omnidirectional mobility. In addition, the proposed wheel consists of single, moldable element and no mechanical components such as bearings and axles, which allows for a reduction in design complexity and potential for decreased production cost and increased robustness.

The proposed wheel is applicable in any situations in which conventional wheels have been used, but in particular, the proposed one will be possible to enhance a mobility performance of an omnidirectional vehicle requiring both high traction performance and omnidirectional motion (i.e. for vehicle towing or for environment such as sloped ground).

\section{ACKNOWLEDGMENT}

This material is based upon work supported in part by the U. S. Army Research Laboratory and the U. S. Army Research Office under contract/grant number W911NF-08-C-0055.
The authors would like to thank Malik Hansen of Boston Dynamics for introducing the idea of multi-material surfaces for robotic mobility.

\section{REFERENCES}

[1] Fujisawa, S., Ohkubo, K., Yoshida, T., Satonaka, N., Shidama, Y., and Yamaura, H., "Improved Moving Properties of an Omnidirectional Vehicle Using Stepping Motor”, Proc of the 36th Conf on Decision \& Control. San Diego, pp.3654-3657, 1997.

[2] Williams, R., Carter, B., Gallina, P., and Rosati, G., "Wheeled Omni-directional Robot Dynamics Including Slip,” Proc of 2002 ASME Design Engineering Technical Conferences, Sep 2002.

[3] Kornylak Corporation, Transwheel ${ }^{\circledR}$, http://www.omniwheel.com/transwheel/transwheel.htm

[4] http://en.wikipedia.org/wiki/Omni_wheel

[5] http://en.wikipedia.org/wiki/Mecanum_wheel

[6] http://www.superdroidrobots.com

[7] AirTrax, http://www.airtrax.com/

[8] Muir, P., and Neuman, C., "Kinematic Modeling for Feedback Control of an Omnidirectional Wheeled Mobile Robot,” Proc. of 1987 IEEE Intl Conf on Robotics and Automation, 1987

[9] Connex500тм Multi-Material 3D Printing System, http://www.objet.com/3D-Printer/Connex500/

[10] Cheng, N., Ishigami, G., Hawthorne, S., Chen, H., Hansen, M., Telleria, M., Playter, R., and Iagnemma, K., "Design and Analysis of a Soft Mobile Robot Composed of Multiple Thermally Activated Joints Driven by a Single Actuator," Proc. of the 2010 IEEE International Conference on Robotics and Automation, May 2010. 